\title{
Implementation of secure email server in cloud environment
}

\begin{abstract}
In the recent virtual communication world, the email services play a vital role as a basic content of heterogeneous networking infrastructure. Whereby, multiple platforms are connecting each other. Mail Server refers to computer performing Mail Transfer Agent functions. MTA is software that delivers electronic mail messages from one computer to another, by using client-server application architecture. MTA implements both sender and receiver portions of SMTP (Simple Mail Transfer Protocol). Postfix is a free and open-source MTA which is fast, easy-to-administrator and provide secure communication over Internet. In this paper we focus on the problem of email contents disclosure, and establish a secure mail server by using Postfix in Linux platform and then implement it into a cloud service provider as IaaS (Infrastructure as a Service). Security is provided by tuning a Transport Layer Security (TLS), and SMTP-AUTH which use Simple Authentication and Security Layer (SASL) as a security mechanism and platform.
\end{abstract}

Keyword: SMTP (Simple Mail Transfer Protocol); Mail transfer agent (MTA); Postfix; IaaS (Infrastructure as a Service); Cloud 